\title{
Evaluation of pulsed electromagnetic field therapy for the treatment of chronic postoperative pain following lumbar surgery: a pilot, double-blind, randomized, sham-controlled clinical trial
}

This article was published in the following Dove Press journal: Journal of Pain Research

\author{
Robert Gordon Sorrell' \\ Jamie Muhlenfeld ${ }^{2}$ \\ John Moffett ${ }^{2}$ \\ Gary Stevens ${ }^{3}$ \\ Steven Kesten ${ }^{4}$ \\ 'Alabama Orthopedic Center, \\ Birmingham, AL, USA; ${ }^{2}$ Regenesis \\ Biomedical Inc., Scottsdale, AZ, USA; \\ ${ }^{3}$ Dynastat Consulting Inc., Bastrop, TX, \\ USA; ${ }^{4}$ SKC Life Sciences, Carlsbad, \\ CA, USA
}

\begin{abstract}
Background: The incidence of chronic postoperative pain following lumbar spinal surgery has increased with the overall increase in the prevalence of lumbar surgery. This study was conducted to evaluate the analgesic effectiveness of pulsed electromagnetic field (PEMF) therapy in subjects with persistent pain following lumbar surgery.
\end{abstract}

Patients and methods: A randomized, double-blind, sham-controlled, multicenter study in 36 subjects with persistent low-back and/or radiating leg pain after lumbar surgery was conducted. Eligible subjects were randomized (1:1:1) to receive one of two doses of therapy (42- $\mu$ s or 38- $\mu \mathrm{s}$ pulse width) or treatment with a sham device. Subjects self-treated twice daily for 60 days. The primary end point was change in pain intensity $(\Delta \mathrm{PI})$ using the Numerical Pain Rating Scale between average baseline (Days -5 to -1 ) and end of treatment (Days 56-60) for lumbar and radiating leg pain. Secondary outcome measures included the Oswestry Disability Index, Beck Depression Inventory-II, Patient Global Impression of Change, and consumption of analgesics. Results: Low-back pain scores for the $42-\mu$ s group decreased by $40.2 \%$ ( $p=0.028)$, compared to $18.6 \%$ for the $38-\mu$ s pulse width group ( $p=0.037$ ) and $25.6 \%$ for the sham group $(p=0.013$ per protocol population). Average leg pain scores decreased by $45.0 \%(42 \mu \mathrm{s}, p=0.009), 17.0 \%$ (38 $\mu \mathrm{s}, p=0.293$ ), and $24.5 \%$ (sham, $p=0.065$ ). The proportion of subjects responding to therapy, time to $30 \%$ reduction in pain scores, and Patient Global Impression of Change were improved with the PEMF 42- $\mu$ s device over the sham control, although results were associated with $p$-values $>0.05$.

Conclusion: PEMF therapy (42- $\mu$ s pulse width) was associated with trends for a reduction in pain, compared to sham treatment. Secondary endpoints were consistent with an overall beneficial effect of the PEMF 42- $\mu$ s pulse width device.

Keywords: failed back surgery syndrome, lumbar surgery, neuropathic pain, pulsed electromagnetic field therapy, neuromodulation, chronic pain, nociceptive pathways

\section{Introduction}

In the USA, it has been estimated that more than 80,000 individuals per year experience chronic pain and/or complications after back surgery. ${ }^{1}$ Persistent or recurrent back and/or leg pain following back surgery - whether associated with laminectomy, discectomy, fusion, or other procedures - is referred to as failed back surgery syndrome (FBSS). ${ }^{2-5}$ Considering patients with FBSS separately from those with similar symptoms but who have not undergone surgery is important, because identification
Correspondence: Steven Kesten SKC Life Sciences, 7476 Capstan Dr., Carlsbad, CA 920II, USA

Tel +l 8779704970

Email skesten0I@gmail.com 
of the underlying cause of pain of patients in this subgroup has therapeutic implications. ${ }^{4,6}$ Patients with persistent pain following back surgery may present with axial (low-back) pain and/or radiating leg pain of varying degrees, ${ }^{2,3,6-8}$ despite having had anatomically satisfactory spinal surgery. ${ }^{4,9,10}$ The underlying cause of FBSS varies by patient. It can be due to mechanical or neuropathic conditions that develop postoperatively, or, in some cases, it can be due to an initial misdiagnosis of the origin of pain, which, as a result, was not addressed by the surgery. $3,5,6,11$

Understanding what constitutes effective management strategies for FBSS remains challenging due to the complex neuropathology of FBSS, ${ }^{12}$ the heterogeneity of underlying causes of FBSS, ${ }^{1,3,4,12}$ and the need for more clinical data to support an evidence-based approach to treatment. ${ }^{13}$ Several types of interventions are used to treat FBSS. Typically, conservative (eg, physical therapy, medication) and/or minimally invasive (eg, epidural injection, adhesiolysis) interventions are used prior to undertaking repeat spinal surgery, which is associated with a decrease in success rate for each subsequent surgery compared with the first surgery. ${ }^{1,3,14}$ Some interventions used to treat FBSS can introduce complications. Pharmaceutical options, for example, are often associated with adverse side effects ${ }^{15-18}$ and have the potential for misuse and addiction, ${ }^{17,19}$ whereas invasive options have been associated with complications such as migration (for implanted devices) and infection. ${ }^{18}$

Alternative approaches for the treatment of chronic pain associated with FBSS include implanted spinal cord stimulators (SCS), ${ }^{18,20,21}$ radiofrequency (RF) ablation (denervation or rhizotomy), ${ }^{2,3,5}$ and, more recently, pulsed electromagnetic field (PEMF) therapy. ${ }^{22}$ Each of these approaches involve electric currents - either delivered via an electrode (SCS or RF ablation) or induced (PEMF) - with the goal of either modifying pain signaling (SCS and PEMF) or ablating nervous tissue (RF ablation) via different methods. There are no data indicating that PEMF devices can induce electrical currents and, possibly, directly modify vital functions. PEMF can, however, alter gene and protein expression. PEMF therapy involves generating a PEMF with a coil located within an applicator pad that is placed adjacent to the area of the body to be treated. In the case of the PEMF device used in the current trial, a carrier frequency $(27.12 \mathrm{MHz})$ is also employed. Biological effects using this approach most likely occur via induction of electrical current within the tissue..$^{23,24}$

Whereas clinical data suggest PEMF therapy is effective for the treatment of postoperative pain in soft tissues, ${ }^{23,25}$ there have been no randomized, double-blind, controlled clinical trials to date on the use of PEMF therapy for FBSS pain. Results of a recent open-label pilot study using PEMF therapy for FBSS pain found clinically meaningful improvements in a subset of study subjects. ${ }^{22}$ As a follow-up to this study, the current study was designed to examine the effect of PEMF therapy in a multicenter, randomized, sham-controlled, double-blind study in patients with chronic pain following lumbar surgery. The hypothesis was that the $42-\mu$ s dose was superior to the sham control. The $38-\mu$ s dose was exploratory to examine whether preliminary laboratory observations with this dose would be associated with clinical effects.

\section{Patients and methods}

\section{Study design}

This was a pilot, multicenter, randomized (1:1:1), doubleblind, sham-controlled, parallel-group study designed to evaluate the analgesic effectiveness of PEMF treatment in patients with persistent pain following lumbar surgery (ClinicalTrials.gov identifier: NCT02416973). ${ }^{51}$ Treatment was administered twice daily over a 60 -day period. The study was conducted at 13 sites in the USA and was designed to enroll 45 patients. Ethics committee approval was obtained from the Quorum Independent Review Board for each site (Table S1) prior to starting the study, and the study was conducted in compliance with the International Standard of Good Clinical Practice (ICH E6-GCP) procedures and the principles of the Declaration of Helsinki (1964).

\section{Study population}

The key inclusion criteria were previous anatomically successful lumbar back surgery for low-back pain, persistent pain in the low back and/or radiating to leg(s) for greater than or equal to 3 months and less than or equal to 36 months following the most recent surgery, stable analgesic dosing regimen for greater than or equal to 30 days, and an mean pain intensity (PI) of greater than or equal to three and less than nine on the 11 point Numerical Pain Rating Scale (NPRS; Table 1). Potential subjects underwent a Screening Visit (Day -14 to Day -10). Written informed consent for study participation was obtained from all subjects.

\section{Study procedures}

Subjects meeting the inclusion and exclusion criteria (Table 1) entered the 10-day run-in phase of the study. During the run-in phase, subjects were trained in the use of the electronic patient-reported outcome (ePRO) diary, and enrolled into the baseline period of the diary. Subjects were randomized if they continued to meet inclusion criteria and none of the exclusion criteria at the end of the run-in period. 
Table I Key inclusion and exclusion criteria

\begin{tabular}{|c|c|}
\hline Inclusion criteria & xclusion criteria \\
\hline $\begin{array}{l}\text { - Age } \geq 22 \text { years } \\
\text { - One or two anatomically successful lumbar back } \\
\text { surgeries for the treatment of low back pain } \\
\text { - Persistent pain in the low back and/or radiating to } \\
\text { leg(s) for } \geq 3 \text { months and } \leq 36 \text { months following the } \\
\text { most recent surgery } \\
\text { - Stable analgesic dosing regimen (including opioid } \\
\text { and nonopioid medication) for } \geq 30 \text { days prior to } \\
\text { the Screening Visit } \\
\text { - Mean pain intensity } \geq 3 \text { and }<9 \text { as measured on the } \\
\text { numeric pain rating scale during the } 10 \text {-day run-in } \\
\text { period } \\
\text { - Completion of a minimum of } 80 \% \text { of the ePRO } \\
\text { diary assessments during the } 10 \text {-day run-in period }\end{array}$ & $\begin{array}{l}\text { - History of more than two lumbar spine surgeries } \\
\text { - Required additional lumbar surgery or surgery of any type prior to Day } 75 \\
\text { - Disc fusion at any level in the most recent surgery } \\
\text { - Any local injection into the lumbar spine within } 30 \text { days prior to the Screening Visit or } \\
\text { within } 6 \text { weeks prior to the Screening Visit with long-acting lidocaine injection products } \\
\text { - Use of systemic corticosteroids within } 2 \text { months of the Screening Visit } \\
\text { - History of an ongoing painful condition that, in the opinion of the investigator, might have } \\
\text { had a confounding influence on the safety or effectiveness analysis } \\
\text { - Implanted pacemaker, defibrillator, neurostimulator, spinal cord stimulator, bone } \\
\text { - } \text { stimulator, cochlear implant, or other implanted device with an implanted metal lead(s) } \\
\text { - } \text { the } 10 \text {-day run run } \text {-in period } \\
\text { - SD around the mean of the mean PI scores during the } 10 \text {-day run-in period was }>2.0 \\
\text { - BMI }>38 \mathrm{~kg} / \mathrm{m}^{2} \\
\text { Previous treatment with the PEMF therapy }\end{array}$ \\
\hline
\end{tabular}

Abbreviations: PI, pain intensity; BMI, body mass index; PEMF, pulsed electromagnetic field; ePRO, electronic patient-reported outcome.

Randomization was in a 1:1:1 ratio to therapy with one of two active PEMF devices (42- $\mu$ s or $38-\mu$ s pulse width) or an identical inactive sham device. The randomization numbers were auto-assigned to subjects from the individual sites' randomization list through a secure interactive web response system as subjects qualified for inclusion into the study. The currently marketed and US Food and Drug Administration (FDA) cleared device (Provant ${ }^{\circledR}$ Therapy System, Regenesis Biomedical, Scottsdale, AZ, USA) utilizes a $42-\mu$ s pulse width. Further experimentation was carried out using cells in culture at Regenesis Biomedical and was based on published literatures. ${ }^{23-25}$ The second active arm was added on the basis of internal laboratorybased research to explore whether a reduction in pulse width would impact efficacy when compared to the $42-\mu \mathrm{s}$ setting. New therapeutic modalities or additional therapeutic regimens other than the study device were not permitted during the study.

Subjects were instructed to self-treat for 30 minutes twice daily (morning and evening; $8 \mathrm{am} \pm 2$ hours and $8 \mathrm{pm}$ \pm 2 hours) for 60 days when at home. Interim Visits were conducted on Days 15, Day 30, and Day 61 to assess safety, concomitant medications, weight, high sensitivity $\mathrm{C}$-reactive protein (hsCRP), the Oswestry Disability Index (ODI), and Beck Depression Inventory II (BDI). Subjects who experienced increased pain during the study were instructed to increase self-administration of their prescribed analgesic(s). Likewise, subjects who experienced decreased pain during the study were instructed to decrease self-administration of their prescribed analgesic(s).

\section{Study devices}

The PEMF device used in the study (Provant ${ }^{\circledR}$ Therapy System) uses a solid-state, fixed-power output radiofrequency generator and transmitter designed to operate at the Federal Communication Commission-authorized medical device frequency of $27.12 \mathrm{MHz}$. Provant is cleared by the FDA for use as an adjunctive treatment for postoperative pain and edema in superficial soft tissues. The therapy system generates a pulsed electromagnetic field using a Class-C RF amplifier. Two pulse durations were evaluated in this study: 42 and $38 \mu \mathrm{s}$. Pulses are repeated every $1,000 \mu \mathrm{s}$, resulting in an output duty cycle of $4.2 \%$ and $3.8 \%$ and requiring an average forward power level of $<5 \mathrm{~W}$. The amount of forward energy emitted from the treatment applicator is preset at 591 $\pm 44 \mathrm{~V} / \mathrm{m}$ at a distance of $5.0 \mathrm{~cm}$ from the radiating surface of the treatment applicator. The doses with the current device were based on the FDA-approved and currently marketed device. Further experimentation was undertaken using cells in culture at Regenesis Biomedical and was based on published literatures. ${ }^{23-25}$

Sham devices were identical to active devices, except that they were deactivated such that no electromagnetic field energy was generated or delivered when the device was turned on. All other functions were identical to that of the active study devices.

\section{ePRO Diary data collection}

All subjects in the study were provided with an ePRO Diary login (DSG Inc, Malvern, PA, USA) to access the diary from any Internet browser for use during the study to allow for 
collection of patient-reported outcomes (average and worst PI, analgesic consumption, and Patient Global Impression of Change [PGIC]). Subjects were instructed by the site, by using a standardized script, to record their PI and analgesic consumption each morning at 8 am ( \pm 2 hours) during the 10-day run-in period (Day -10 to Day -1 ). Furthermore, subjects were instructed to record their ePRO data (PI, analgesic consumption, and PGIC) immediately following each morning treatment for 60 days (Days 1-60), and continue to collect ePRO data for 15 days following completion of treatment each morning at 8 am ( \pm 2 hours). Subjects were prompted to report the daily mean and worst PI for their lumbar back pain and/or radiating leg pain separately, analgesic consumption, and PGIC every 7 days. For analgesic consumption, subjects were prompted to report their analgesic consumption (opioid and nonopioid) prescribed for the treatment of the subject's lumbar back and/or radiating leg pain over the preceding 24 hours.

\section{Outcome measurements}

\section{Pain intensity}

The PI was self-reported by subjects using the validated 11-point NPRS. ${ }^{26,27}$ For PI analyses, mean PI scores were calculated for every 5-day interval of the trial period (ie, a total of 16 intervals for per protocol [PP] subjects) for each of the four PI score categories reported (ie, worst back PI, mean back PI, worst leg PI, mean leg PI). The baseline PI was defined as the mean PI for the last 5 days of the 10-day run-in period, and end-of-treatment PI was defined as the mean PI for the last 5 days of the 60-day treatment period.

\section{Responder analyses}

For the prespecified responder analysis, subjects reporting a two-point reduction in PI or a $30 \%$ decrease in PI from baseline were considered responders. ${ }^{29}$

\section{Area under the curve}

Post hoc area under the curve (AUC) analyses were conducted to assess PI changes over time for all randomized subjects without imputation. AUC analyses were undertaken using SAS software. ${ }^{28}$

\section{Change from baseline PI AUC}

The analysis was conducted using SAS software by integration.

\section{Analgesic consumption}

Subjects self-reported their consumption of analgesic tablets by recording the number of pills of each medication consumed in the preceding 24 hours in the ePRO diary. Analgesic medications refer to medications prescribed and administered for the treatment of pain and included opioids, nonsteroidal anti-inflammatory agents, antidepressants, and muscle relaxants. The analysis on analgesic consumption was based on the number and percentage change in the number of pills taken at each 5-day period compared with the 10-day run-in period.

\section{Other patient-reported outcomes}

The PGIC is a seven-point validated categorical scale of overall change in status. ${ }^{29,30}$

The ODI (version 2.1a) consists of a self-reported questionnaire in categorical format that assesses a subject's level of disability for common activities such as walking, sleeping, and personal care. ${ }^{31}$

Symptoms of depression were assessed using the BDI, version II. ${ }^{32}$ A score of 13 or less is considered indicative of minimal depression, whereas a score of 29 or greater indicates severe depression.

\section{High-sensitivity C-reactive protein}

The hsCRP levels were assayed using blood samples obtained at the Enrollment and Interim Visits as a biomarker of inflammation. ${ }^{33,34}$

\section{Safety}

Safety was monitored through the collection of adverse events, including serious adverse events. The Medical Dictionary for Regulatory Activities coding dictionary was used to categorize adverse events.

\section{Statistical analyses}

Analysis of the primary endpoint (PI) was conducted on the intent-to-treat (ITT) population using the last observation carried forward (LOCF) imputation method, together with a median and quartile imputation. A sensitivity analysis was undertaken on the primary endpoint using a PP population. The PP population was identified prior to the unblinding of the study data and was defined on the basis of subjects that completed 60 days of treatment and did not have significant protocol deviations. The quartile imputation was a worstcase imputation that used the 25 th percentile of the treatment group to impute values for the active groups and used the 75 th percentile for imputation of values in the sham group. There were no imputations carried out on missing data for the secondary endpoints. There were no adjustments for multiple testing. Adverse events were summarized by number and incidence. The sample size of 15 patients per group and 
45 in total was a sample of convenience for this study. No power calculations were conducted.

Statistical tests to evaluate the significance of withingroup (WG) and between-group (BG) differences in PI scores were conducted in SAS software. WG and BG differences were tested using one-way analysis of variance. Data were plotted using Sigma Plot 13.0.

\section{Results}

In total, 69 subjects were screened at 13 sites. Of the 69 screened, 52 subjects entered the 10-day run-in period. Eleven subjects were found to be ineligible during the run-in period, leaving 41 eligible subjects who were enrolled in the study and who received at least one treatment (ITT population). Of the 41 subjects in the ITT population, 36 subjects completed the 60-day treatment protocol (PP population). Of the five ITT subjects who withdrew early, three subjects (42- $\mu$ s group) withdrew on Days 9, 32, and 36 due to an adverse event, one subject (38- $\mu$ s group) withdrew on Day 7 due to personal reasons, and one subject (sham group) withdrew on Day 15 due to lack of efficacy (Figure 1). The study was closed to enrollment prior to achieving the planned enrollment (45 subjects) in order to allow for inclusion of study results in a planned regulatory submission.

\section{Demographics}

Demographics and clinical characteristics at baseline were generally similar between study groups (Table 2). There was a slightly higher proportion of males in the $42-\mu$ s group $(62 \%, 8 / 13)$ relative to the sham group $(36 \%, 5 / 14)$. The

Table 2 Subject demographics and baseline characteristics

\begin{tabular}{|c|c|c|c|c|}
\hline & Sham & 38-ps group & 42-ps group & p-value \\
\hline Gender, n (\%) & & & & 0.46 \\
\hline Male & $5(36.0)$ & $7(50.0)$ & $8(62.0)$ & \\
\hline Female & $9(64.0)$ & $7(50.0)$ & $5(36.0)$ & \\
\hline Age (years) & $50.6(12.2)$ & $51.2(12.3)$ & $56.0(14.3)$ & 0.59 \\
\hline Height $(\mathrm{cm})$ & $170(12.2)$ & $173(15.7)$ & $169(10.7)$ & 0.69 \\
\hline Weight (kg) & $85(19.3)$ & $86.2(22.5)$ & $87.5(13.7)$ & 0.94 \\
\hline BMI $\left(\mathrm{kg} / \mathrm{m}^{2}\right)$ & $29.3(5.1)$ & $28.6(5.8)$ & $30.7(4.5)$ & 0.56 \\
\hline $\begin{array}{l}\text { Time since } \\
\text { surgery (months) }\end{array}$ & I5.I (10.4) & $12.6(8.6)$ & $12.7(7.6)$ & 0.70 \\
\hline $\mathrm{PI}$, lumbar & $5.1(1.0)$ & $5.5(1.6)$ & 4.9 (1.9) & $0.4 I$ \\
\hline $\mathrm{PI}$, radiating leg & $4.3(1.8)$ & $5.4(1.6)$ & $5.1(1.8)$ & 0.83 \\
\hline ODI & $33.8(14.7)$ & 39.7 (17.9) & $32.4(9.6)$ & 0.48 \\
\hline BDI (units) & $10.1(6.74)$ & $12.4(5.57)$ & $9.7(6.55)$ & 0.80 \\
\hline hsCRP (mg/L) & $2.3(2.1)$ & $2.1(2.6)$ & 0.56 & 0.5857 \\
\hline
\end{tabular}

Note: Data are reported for ITT population/and recorded as mean ( \pm SD) unless otherwise specified.

Abbreviations: $\mathrm{n}$, number of subjects; $\mathrm{PI}$, pain intensity; ODI, Oswestry Disability Index; BDI, Beck Depression Inventory; hsCRP, high-sensitivity C-reactive protein; ITT, intent-to-treat; BMI, body mass index.

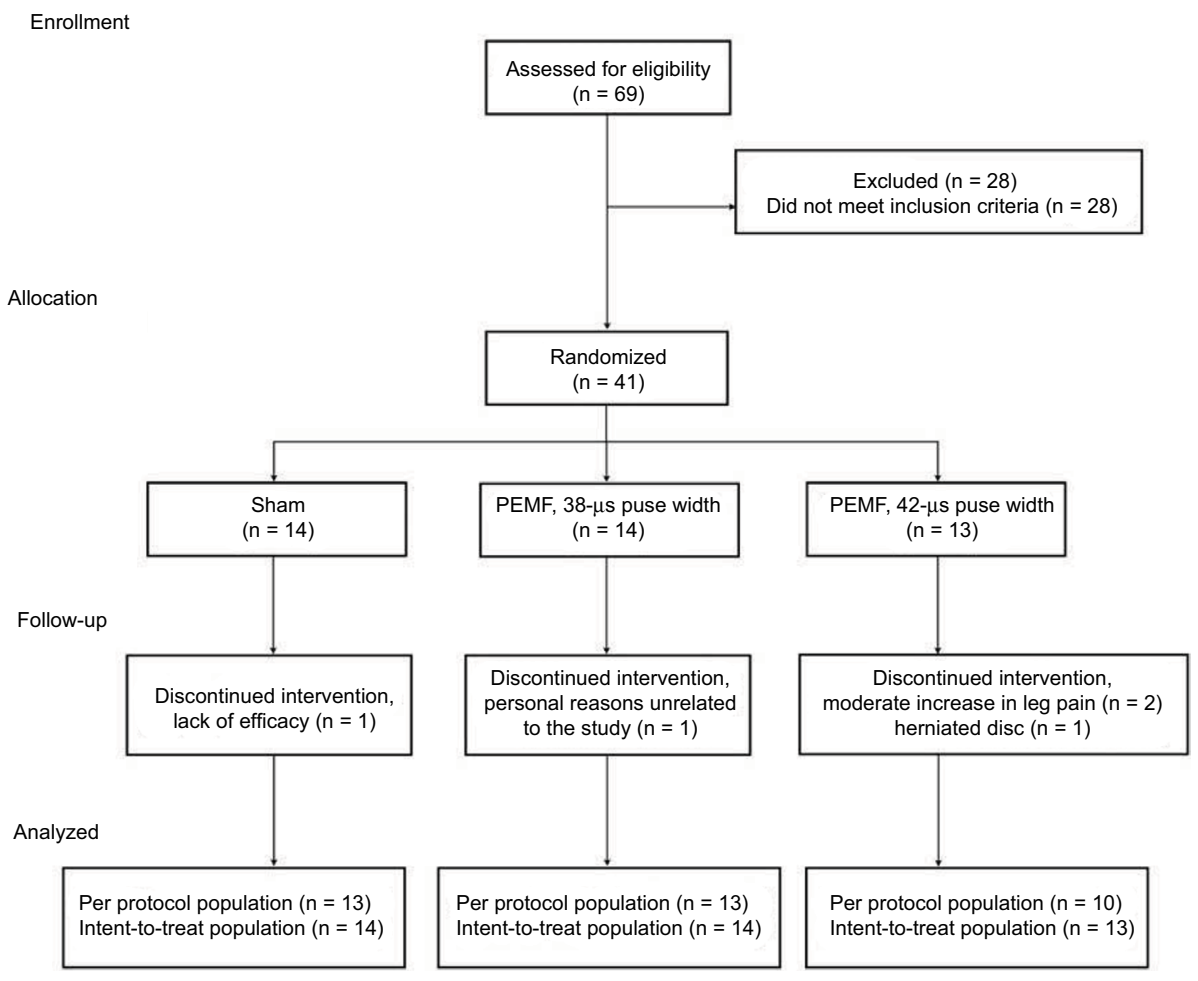

Figure I Study flow chart and patient profile.

Abbreviations: $n$, number of subjects; PEMF, pulsed electromagnetic field. 
mean duration of time from the most recent surgery was 12.7 , 12.6, and 15.1 months for the $42-\mu \mathrm{s}, 38-\mu \mathrm{s}$, and sham groups, respectively. Baseline data were generally similar for each of the study groups. The majority of subjects reported both lumbar (40/41) and radiating leg (35/41) pain. Mean BDI scores were low at baseline for all study groups. In terms of concurrently used interventions during the study, $83 \%$ of ITT subjects reported participating in regular at-home stretching or exercise, and 7\% of ITT subjects received inoffice physical therapy.

\section{Compliance}

The mean total duration of device usage per subject during the study was $57.2 \pm 4.4,49.7 \pm 14.5$, and $46.7 \pm 17$ hours, respectively, for the $42-\mu \mathrm{s}, 38-\mu \mathrm{s}$, and sham groups for the PP population, based on the device usage meter data.

\section{Pain intensity}

The mean end-of-treatment PI was lower for the 42- $\mu$ s group than for the other study groups, for both lumbar and radiating leg pain (Figure 2A and B, Table 3 ). The primary endpoint
A



B Baseline vs Days $56-60$ pain scores (radiating leg)

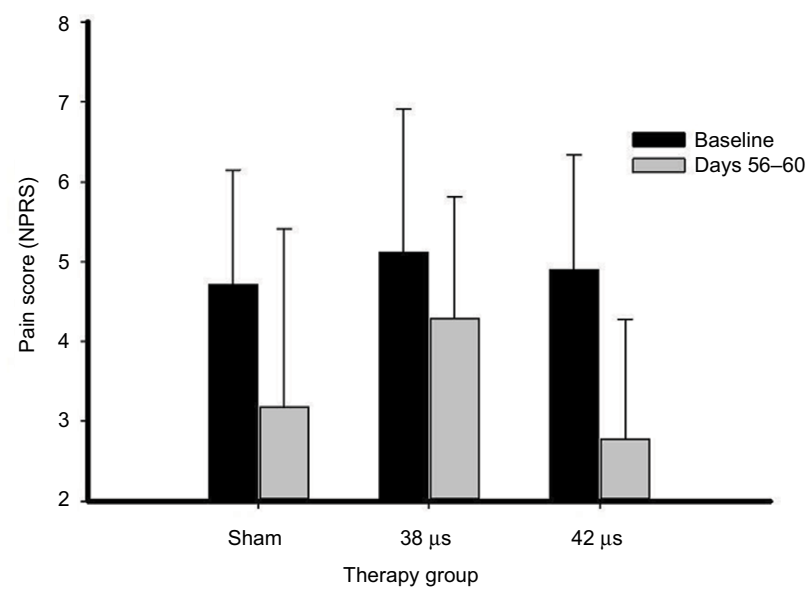

Figure 2 Mean pain scores at the end of treatment (Days 56-60) compared with baseline.

Notes: (A) Lumbar pain. (B) Radiating leg pain. Statistical analyses were conducted using one-way analysis of variance and show the within-group $p$-values. Abbreviation: NPRS, numerical pain rating scale.

Table 3 Primary outcome measure at 60 days

\begin{tabular}{|c|c|c|c|}
\hline & $\begin{array}{l}\text { Sham } \\
\text { group } \\
(n=14)\end{array}$ & $\begin{array}{l}38-\mu s \\
\text { group } \\
(n=14)\end{array}$ & $\begin{array}{l}\text { 42- } \mu s \\
\text { group } \\
(n=10)\end{array}$ \\
\hline Subjects completed study, $\mathrm{n}$ & 13 & 13 & 9 \\
\hline Compliance, hours $( \pm S D)$ & $46.7(16.96)$ & $49.7(|4.5|)$ & $57.2(4.38)$ \\
\hline Lumbar NPRS, mean PI, baseline, Day 0 (SD) & $5.1(0.96)$ & $5.5(1.79)$ & $4.9(1.94)$ \\
\hline Lumbar NPRS, mean PI, end of treatment, Day 60 (SD) & $3.8(1.58)$ & $4.2(1.63)$ & $2.9(1.66)$ \\
\hline Lumbar mean Pl, absolute mean change & -1.3 & -1.3 & -2 \\
\hline Lumbar \% change from baseline in mean PI at Day 60 (SD) & $-25.6(24.8 I)$ & $-18.6(39.47)$ & $-40.2(26.35)$ \\
\hline \multirow[t]{2}{*}{ Lumbar mean $\mathrm{Pl} 95 \% \mathrm{Cl}$} & $(39.1,-12.1)$ & $(-40.1,2.9)$ & $(-57.4,-23.0)$ \\
\hline & $(n=12)$ & $(n=10)$ & $(n=8)$ \\
\hline Radiating leg NPRS, mean PI, baseline, Day 0 (SD) & $4.3(1.78)$ & $5.4(1.6)$ & $5.1(1.83)$ \\
\hline Radiating leg NPRS, mean PI, end of treatment, Day 60 (SD) & $3.3(2.29)$ & $4.3(1.6)$ & $2.8(1.60)$ \\
\hline Radiating leg mean $\mathrm{PI}$, absolute mean change & -1 & -1.1 & -2.3 \\
\hline Radiating leg \% change from baseline in mean Pl at Day 60 (SD) & $-24.5(33.79)$ & $-17.0(31.15)$ & $-45.0(29.39)$ \\
\hline Radiating leg mean PI 95\% Cl & $(-44.5,-4.5)$ & $(-36.3,2.3)$ & $(-65.4,-24.6)$ \\
\hline
\end{tabular}

Abbreviations: PI, pain intensity; NPRS, numerical pain rating scale. 
which was the percentage change from baseline in mean PI scores through Day 60 (end-of-treatment) summaries were conducted on the ITT populations using the LOCF method for imputation of missing data along with a median and quartile imputation. The mean lumbar PI for the $42-\mu \mathrm{s}$ group decreased by 1.1 units $(-17.9 \%)$ compared with 1.4 units $(-24.9 \%)$ in the $38-\mu$ s group and 1 unit $(-21.1 \%)$ in the sham group. For the mean radiating leg PI, the $42-\mu \mathrm{s}$ group decreased by 1.3 units (-20.2\%), compared with 1.1 units $(-21.3)$ in the $38-\mu$ s group and 0.9 units $(-26.1 \%)$ in the sham group. A sensitivity analysis was conducted on the PP population and showed a similar trend, with a greater decrease in both lumbar and radiating leg PI reported for the $42-\mu$ s group compared to the $38-\mu$ s group or the sham group. Mean lumbar PI for the $42-\mu$ s group decreased by 2 units $(40.2 \%, p=0.028)$, compared to a decrease of 1.3 units $(18.6 \%, p=0.037)$ and 1.3 units $(25.6 \%, p=0.013)$ for the $38-\mu$ s and sham groups, respectively (Figure $3 \mathrm{~A}$ and $B$, Table 3). Figure 4A and B shows pain scores normalized to baseline values. For mean radiating leg PI, a decrease of 2.3 units was observed at the end of treatment relative to baseline for the $42-\mu$ s group $(45.0 \%, p=0.009)$, compared to a decrease of 1.1 units for the 38 - $\mu$ s group $(17.0 \%, p=$ $0.293)$, and a decrease of 1 unit $(24.5 \%, p=0.065)$ for the sham group (Figure 3B, Table 3).

The change in PI was further evaluated by a prespecified responder analysis as well as by a post hoc AUC analysis and time-to-event analysis for PI over the course of the study
(Table 4). In all cases, results were consistent with those obtained for the primary endpoint. For the responder analysis, subjects who experienced a reduction in PI of 2 points or $30 \%$ (mean final PI vs average baseline PI) were considered responders. A two-point or $30 \%$ reduction in the pain scores is considered a clinically meaningful change in $\mathrm{PI}^{27,29}$ and is associated with not needing rescue medication and ratings of "much" or "some" improvement. ${ }^{29}$ Based on the latter criterion, $55.6 \%$ and $53.9 \%$ of PP subjects in the $42-\mu$ s and $38-\mu$ s groups, respectively, were responders based on change in lumbar PI, compared to $30.8 \%$ of PP subjects in the sham group (Table 4). None of the subjects reported a two-point decrease in PI without a $30 \%$ decrease in PI. In addition, a $50 \%$ or greater reduction in lumbar PI was observed in $44.4 \%$ of subjects in the $42-\mu$ s group, compared with no subjects in the $38-\mu$ s group, and $15.4 \%$ of subjects in the sham group. A reduction in PI of $50 \%$ or greater is considered to represent substantial improvement, corresponding to a rating of "very much improved." ${ }^{29}$ Similar trends were observed for the responder analyses of mean radiating leg PI (Table 3). The median time to $30 \%$ response for both lumbar and radiating leg PI was shorter in the 42- $\mu$ s group (21-25 days and 21-25 days, respectively) compared with the other study groups: 61-65 and 41-45 days, respectively in the sham group, and 35-40 and 41-45 days for the 38- $\mu$ s group (Table 4).

Post hoc pain AUC analyses were conducted in order to utilize all data for all randomized subjects without imputation. ${ }^{28}$ Analyses were conducted without and with adjustments
A

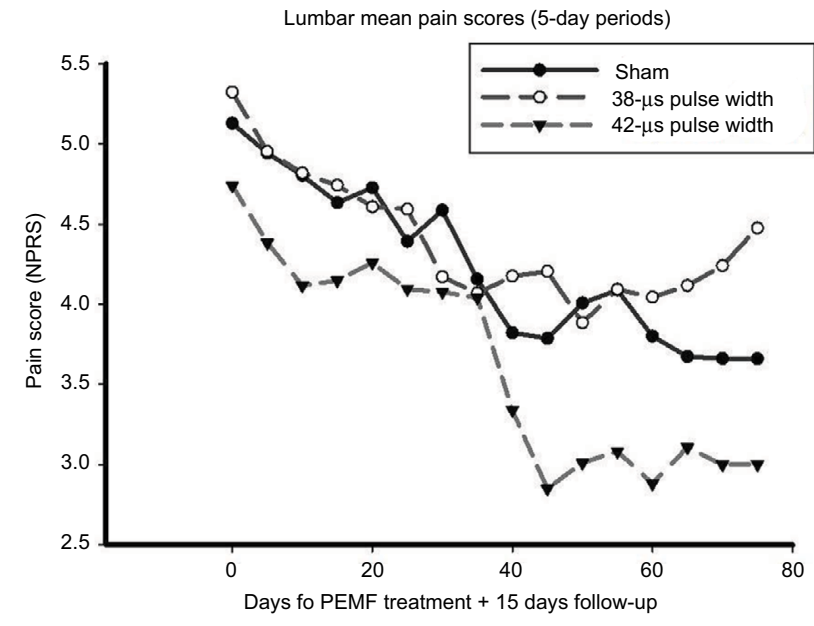

B

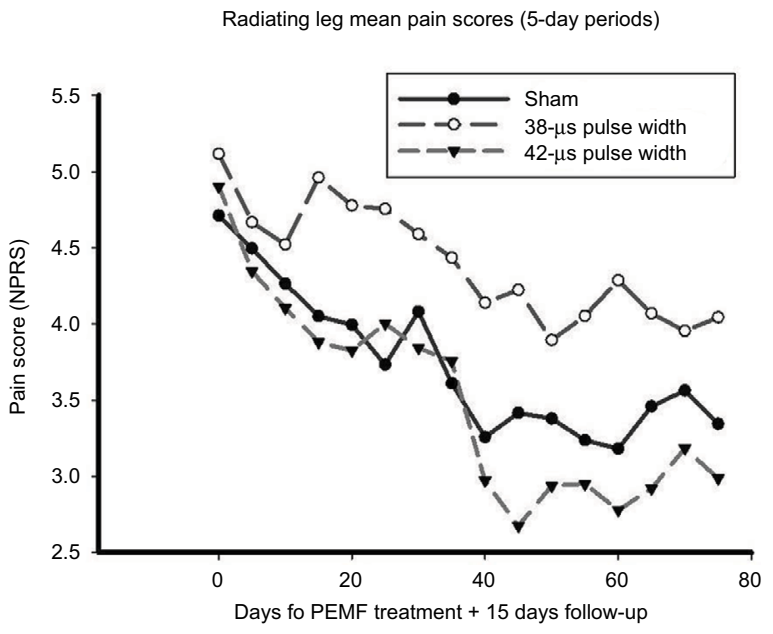

Figure 3 Mean pain scores over time.

Notes: (A) Lumbar pain. (B) Radiating Leg pain. Mean pain scores are for consecutive 5-day intervals throughout the study.

Abbreviations: NPRS, numerical pain rating scale; PEMF, pulsed electromagnetic field. 
A

Lumbar pain scores normalized to baseline (\%)

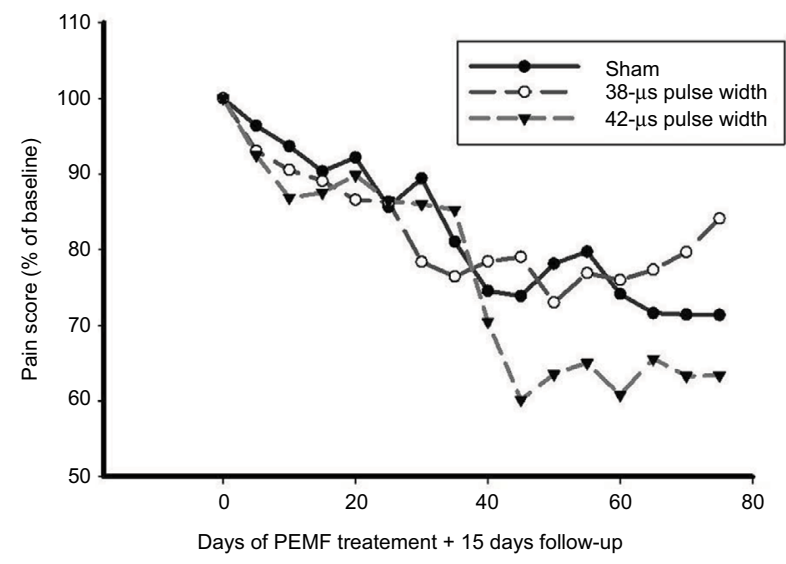

B

Radiating leg pain scores normalized to baseline (\%)

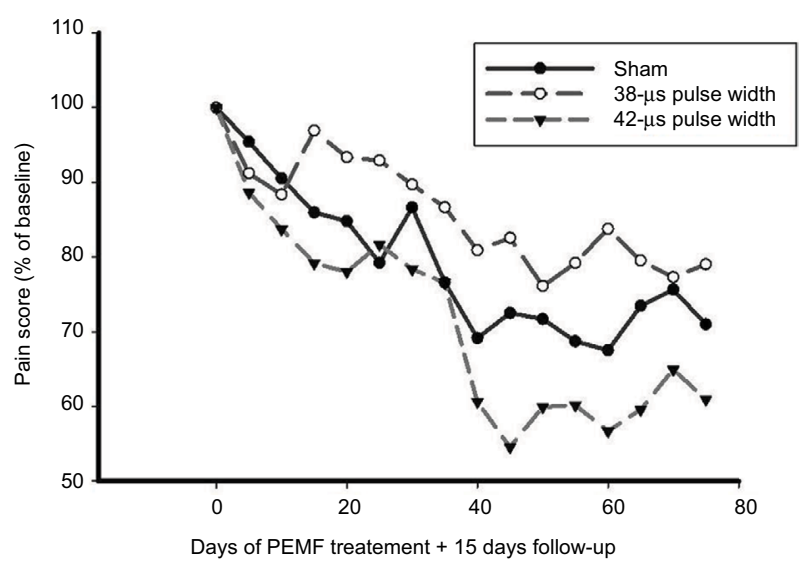

Figure 4 Mean pain scores over time normalized to baseline.

Notes: (A) Lumbar pain. (B) Radiating Leg pain. For each study group, the mean pain scores were normalized to baseline pain scores, and plotted as a percentage of baseline. Average pain scores are for consecutive 5-day intervals throughout the study. For each study group, data are expressed as a percentage of the mean baseline pain score for that study group.

Abbreviation: PEMF, pulsed electromagnetic field.

for baseline differences. The unadjusted AUC for mean lumbar PI was numerically (24.5\%) less for ITT subjects in the $42-\mu$ s group $(186.5 \pm 84.2)$ compared with the sham group (246.9 $\pm 74.1 ; p=0.694$, Table 4$)$. Consistent with the primary endpoint, findings were similar for AUC between the sham and the $38-\mu$ s groups $(251.4 \pm 120.9)$. For mean radiating leg PI, the AUC for ITT subjects in the $42-\mu$ s group $(173.5 \pm 70.8)$ was $19.3 \%$ less than that for the sham group (AUC: $238.1 \pm 101.1 ; p=0.231$ ). The mean radiating leg AUC for the $38-\mu$ s group was similar to that for the sham group (Table 4).

The analysis of AUC adjusted for baseline differences for the mean change from baseline PI showed a $130.3 \%$ decrease in this measure for radiating leg PI in the $42-\mu \mathrm{s}$ group (-69.7), which was larger than the same measure for the sham group (-29.4). Furthermore, results for the $38-\mu \mathrm{s}$ group were better than those observed for the sham group $(-48.2)$ although no results for any of the groups reached statistical significance (Table 4).

The median time to $30 \%$ reduction in pain scores was

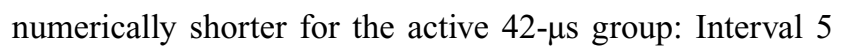
(Days 21-25) compared to Interval 9 (Days 41-45) for the sham group for lumbar pain ( $p=0.33$, Figure $5 \mathrm{~A})$. For radiating leg pain, in the $42-\mu$ s group, the median time to response was Interval 5 (Days 21-25), compared to Interval 13 (Days $61-65$ ) for the sham group ( $p=0.32$, Figure 5B). The changes for the 38- $\mu$ s group are somewhat between that of the $42-\mu \mathrm{s}$ and the sham groups (data not shown).

\section{Secondary endpoints}

To monitor possible effects of the therapy on inflammation, levels of the inflammatory marker hsCRP were assessed at baseline and at Day 61 following the end of treatment. For the $42-\mu$ s group, the mean hsCRP level was lower at Day 61 compared to baseline (Tables 2 and 4), whereas, conversely, the mean hsCRP levels for the sham and 38- $\mu$ s groups were higher at Day 61 compared with baseline (Tables 2 and 4) although BG differences were associated with $p>0.05$. No relevant BG differences were observed for ODI and BDI (Table 4). On Day $60,75 \%$ of subjects in the $42-\mu$ s group reported some level of improvement based on data collected using the PGIC questionnaire, whereas 58.2\% and $44.4 \%$ of sham and 38- $\mu$ s group subjects, respectively, reported some level of improvement. With regard to analgesic use, all groups showed minor decreases in analgesic use. For the sham group, the median decrease in pill count at Days 56-60 was $-0.67 \pm 1.38, \mathrm{n}=12$. For the $38-\mu \mathrm{s}$ and $42-\mu \mathrm{s}$ pulse groups, the decrease in pill counts was $-0.34 \pm 0.80$, $\mathrm{n}=13$ and $1.06 \pm 1.13, \mathrm{n}=9$, respectively. Changes in the secondary endpoints, although directionally in favor of active treatment, may not necessarily be considered clinically significant.

\section{Safety}

Adverse events were reported in six subjects (46.2\%) in the $42-\mu$ s group, three subjects $(21.4 \%)$ in the $38-\mu$ s group, and five subjects (35.7\%) in the sham group. None of the events 
Table 4 Secondary outcome measures at 60 days

\begin{tabular}{|c|c|c|c|}
\hline & Sham group & 38-ps group & 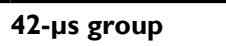 \\
\hline Responder analysis: percentage of patients with a $30 \%$ reduction in NPRS & $(n=13)$ & $(n=13)$ & $(n=9)$ \\
\hline \multicolumn{4}{|l|}{ (Days 56-60) } \\
\hline \multicolumn{4}{|l|}{ Lumbar $(\%$ of $n)$} \\
\hline $30 \%$ change in mean $\mathrm{PI}(\%)$ & 30.77 & 46.15 & 55.56 \\
\hline $30 \%$ change in worst $\mathrm{PI}(\%)$ & 23.08 & 38.46 & 55.56 \\
\hline Radiating leg (\% of $n$ ) & $(n=12)$ & $(n=10)$ & $(n=8)$ \\
\hline $30 \%$ change in mean $\mathrm{PI}(\%)$ & 41.67 & 40.00 & 50.00 \\
\hline $30 \%$ change in worst $\mathrm{PI}(\%)$ & 41.67 & 40.00 & 37.50 \\
\hline Percentage change from baseline in worst PI (n) & $(n=13)$ & $(n=13)$ & $(n=9)$ \\
\hline Lumbar NPRS, worst PI, baseline, Day 0 (SD) & $6.5(1.43)$ & $7.0(1.75)$ & $6.3(1.46)$ \\
\hline Lumbar NPRS, worst PI, end of treatment, Day 60 (SD) & $5.4(1.93)$ & $5.7(2.39)$ & $4.3(2.28)$ \\
\hline Lumbar worst PI mean change & -1.1 & -1.3 & -2 \\
\hline Lumbar \% change from baseline in worst PI at Day 60 (SD) & $-17.9(24.22)$ & $-19.6(24.35)$ & $-33.8(31.89)$ \\
\hline \multirow[t]{2}{*}{ Lumbar worst $\mathrm{Pl} 95 \% \mathrm{Cl}$} & $(-3||,-4.7)$. & $(-32.8,-6.4)$ & $(-54.6,-13.0)$ \\
\hline & $(n=12)$ & $(n=10)$ & $(n=8)$ \\
\hline Radiating leg NPRS, worst PI, baseline, Day 0 (SD) & $5.9(2.38)$ & $6.5(1.98)$ & $6.5(1.64)$ \\
\hline Radiating leg NPRS, worst PI, end of treatment, Day 60 (SD) & $4.9(3.00)$ & $5.3(2.05)$ & $4.4(1.73)$ \\
\hline Radiating leg worst PI mean change & -1 & -1.2 & -2.1 \\
\hline Radiating leg \% change from baseline in worst PI at Day 60 (SD) & $-19.2(37.07)$ & $-14.6(36.45)$ & $-29.5(27.57)$ \\
\hline Radiating leg worst PI 95\% Cl & $(-4 I .1,2.7)$ & $(-37.2,8.0)$ & $(-48.6,-10.4)$ \\
\hline Oswestry Disability Index & $(n=13)$ & $(n=13)$ & $(n=10)$ \\
\hline Mean baseline (SD) & $33.8(14.73)$ & $39.7(17.94)$ & $32.4(9.65)$ \\
\hline Mean visit Day 6I (SD) & $28.8(13.85)$ & $33.1(18.97)$ & $27.0(13.64)$ \\
\hline Percentage difference $(95 \% \mathrm{Cl})$ & $-14.9(-24.9,-4.9)$ & $-17.7(-33.5,-1.9)$ & $-15.2(-42.0,11.6)$ \\
\hline Beck Depression Inventory (responders with $>5$ point decrease) & $23.08 \%$ & $30.77 \%$ & $10 \%$ \\
\hline hsCRP levels (mg/L) & $(n=13)$ & $(n=I I)$ & $(\mathrm{n}=9)$ \\
\hline Baseline (SD) & $2.3(2.11)$ & $2.1(2.59)$ & $3.2(2.72)$ \\
\hline Day $6 \mathrm{I}$ (SD) & $4.5(6.27)$ & $2.8(2.60)$ & $2.7(2.32)$ \\
\hline Difference $(95 \% \mathrm{Cl})$ & $2.2(-1.3,5.7)$ & $0.7(-0.4,1.8)$ & $-0.5(-1.8,0.8)$ \\
\hline \multicolumn{4}{|l|}{ Patient global impression of change (\% patients, Day 60 ) } \\
\hline Minimally worse & $0(0.0)$ & I (II.I) & $0(0.0)$ \\
\hline No change & $5(45.5)$ & $4(44.4)$ & $2(25.0)$ \\
\hline Minimally improved & $4(40.0)$ & $2(22.2)$ & $3(37.5)$ \\
\hline Much improved & I (9.I) & $2(22.2)$ & $3(37.5)$ \\
\hline Very much improved & $\mathrm{I}(9.1)$ & $0(0.0)$ & $0(0.0)$ \\
\hline Time to $30 \%$ decrease in mean $\mathrm{PI}$, days & $(n=14)$ & $(n=14)$ & $(n=12)$ \\
\hline Lumbar $(95 \% \mathrm{Cl})$ & $22.0(12.00,41.00)$ & $19.00(6.00,32.00)$ & $8.00(6.00,32.00)$ \\
\hline Radiating leg $(95 \% \mathrm{Cl})$ & $10.00(1.00,23.00)$ & $16.00(2.00,36.00)$ & $6.00(3.00,14.00)$ \\
\hline Time to $30 \%$ decrease in worst $\mathrm{PI}$, days & $(n=14)$ & $(n=14)$ & $(n=12)$ \\
\hline Lumbar $(95 \% \mathrm{Cl})$ & $22.0(3.00,55.00)$ & $17.00(8.00,25.00)$ & $8.00(3.00,21.00)$ \\
\hline Radiating leg $(95 \% \mathrm{Cl})$ & $20.00(2.00,43.00)$ & $32.00(3.00,32.00)$ & $9.00(1.00,30.00)$ \\
\hline Post hoc analysis-pain (AUC) & $(n=14)$ & $(n=14)$ & $(n=12)$ \\
\hline Lumbar mean integrated pain (SD) & $246.9(74.07)$ & $251.4(120.86)$ & I86.5 (84.17) \\
\hline$(95 \% \mathrm{Cl})$ & $(208.1,285.7)$ & $(188.1,314.7)$ & $(138.9,234.1)$ \\
\hline Lumbar mean integrated change from baseline (SD) & $-45.1(54.03)$ & $-50.7(71.38)$ & $-56.8(88.80)$ \\
\hline$(95 \% \mathrm{Cl})$ & $(-73.4,-16.8)$ & $(-88.1,-13.3)$ & $(-107.0,-6.6)$ \\
\hline Radiating leg mean integrated pain (SD) & $215.1(101.05)$ & $238.1(126.62)$ & I 73.5 (70.77) \\
\hline$(95 \% \mathrm{Cl})$ & $(160.2,270.0)$ & $(163.3,312.9)$ & $(131.7,215.3)$ \\
\hline Radiating leg mean integrated change from baseline (SD) & $-29.4(55.67)$ & $-48.2(86.28)$ & $-69.7(91.10)$ \\
\hline$(95 \% \mathrm{Cl})$ & $(-59.7,0.9)$ & $(-99.2,2.8)$ & $(-123.5,-15.9)$ \\
\hline
\end{tabular}

Notes: Data reported as mean \pm SD (device usage, end-of-treatment PI and ODI scores, end-of-treatment hsCRP levels, \% change in PI), mean with $95 \%$ Cls (time to $30 \%$ decrease in PI, \% change in ODI, change in hsCRP), percentage of subjects analyzed (responder analyses, PGIC, BDI), and mean AUC score with percentage change from baseline (post hoc AUC). Summary statistics reported are for the PP population, except for time to $30 \%$ decrease in PI and AUC analyses, which are reported for the ITT population. For PP population analyses, only subjects reporting data at end of treatment were included in the analysis. Some subjects reported only lumbar or radial pain. Data for all of these subjects are included in the summary statistics for lumbar and radiating leg PI scores reported. For percentage change from baseline for PI, AUC, and ODI, improvement is denoted by a negative value. For baseline scores, see Table 4. For all analyses using PI scores, mean PI scores were used. End-of-treatment PI scores refers to the average of scores for Days 56-60. End of treatment for ODI and hsCRP refers to the Day 6I Interim Visit. PGIC data are reported for Day 60. BDI data are reported for Day 60.

Abbreviations: PI, pain intensity; AUC, area under the curve; ODI, Oswestry Disability Index; PGIC, Patient Global Impression of Change; BDI, Beck Depression Inventory; hsCRP, high-sensitivity C-reactive protein; $n$, number of subjects; PP, per protocol. 
A

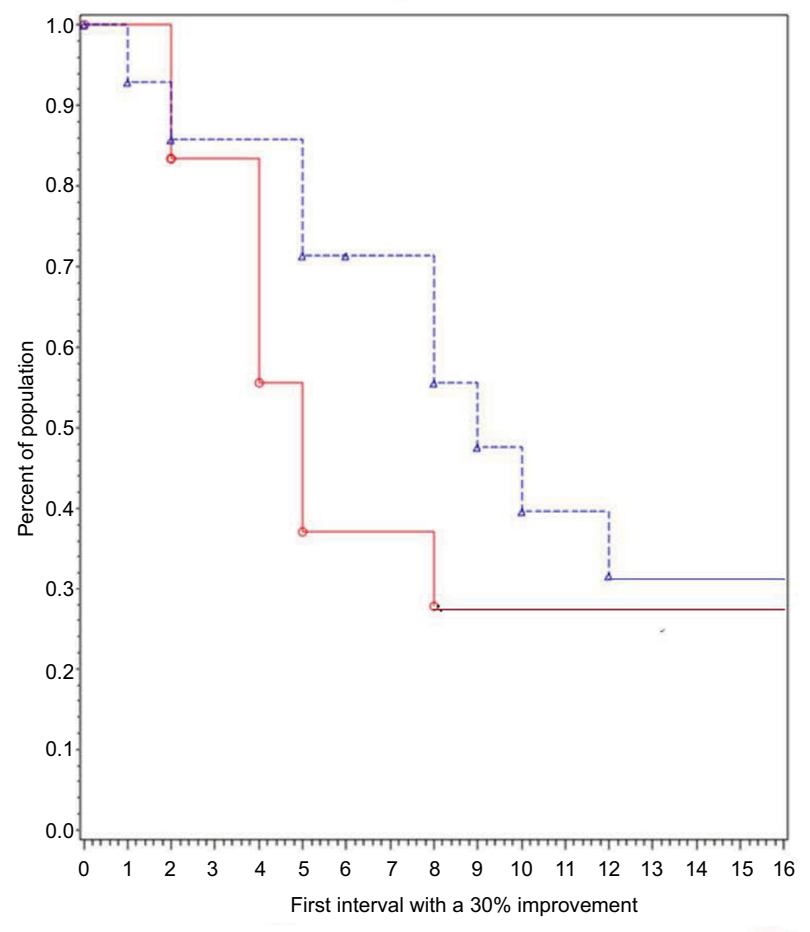

B

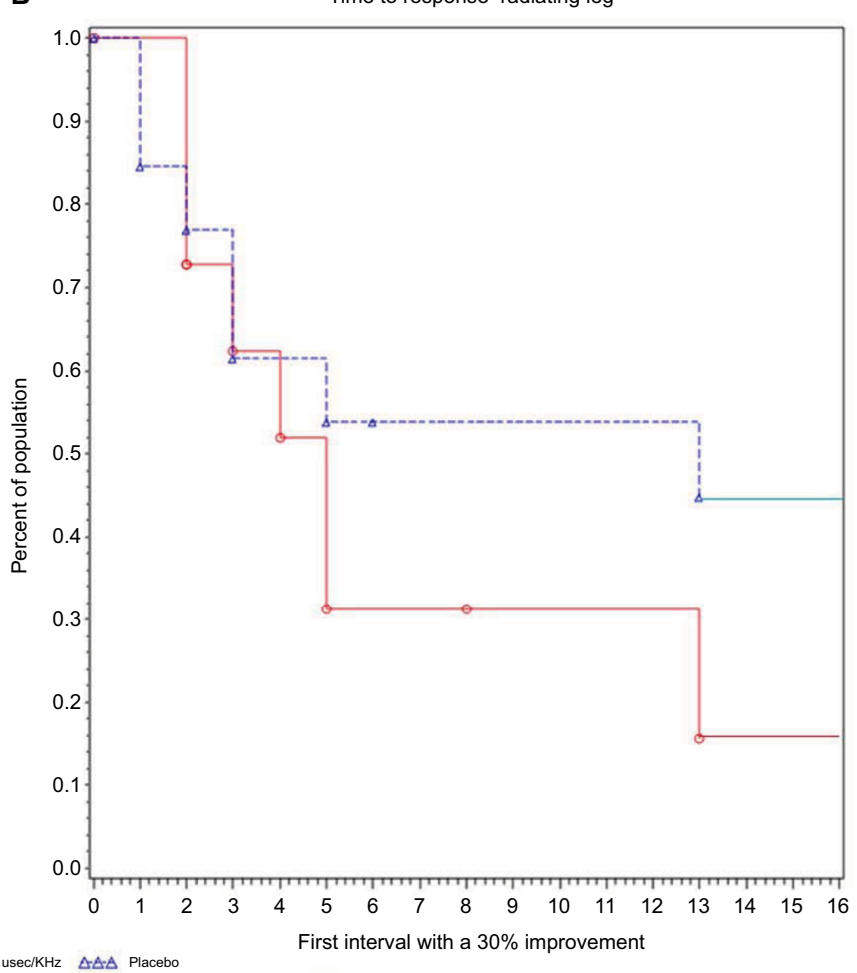

Figure 5 Time to $30 \%$ reduction in pain score is the time to response. Figures show the time to $30 \%$ reduction, which was evaluated using Kaplan-Meier statistics. Notes: (A) Lumbar pain, median time to response-Interval 5 ( 2 I-25 days) for $42 \mu$ s and Interval 9 (4I-45 days) for sham ( $p=0.33$, log-rank test). (B) Radiating leg pain, median time to response-Interval 5 (2I-25 days) for $42 \mu$ s and Interval I3 (6I-65 days) for sham ( $p=0.32$, log-rank test).

were considered serious. Musculoskeletal disorders were the most common adverse event reported, with 5 in the $42-\mu \mathrm{s}$ group (back pain [2], disc protrusion [1], and extremity pain [2]), 0 in the 38- $\mu$ s group, and 1 (back pain) in the sham group. All of the other adverse events were reported by no more than one subject within a treatment group.

\section{Discussion}

Diagnosing FBSS (also called Postoperative Persistent Syndrome, ie, POPS $)^{4}$ and understanding its etiology is complex and has led to challenges in treatment. ${ }^{3}$ Interventional procedures such as epidural injections, ablation, or finally surgical revision have met with limited success. ${ }^{35,36}$ Conservative treatments such as physical therapy, acupuncture, and behavioral therapies are noninvasive and, therefore, are commonly used treatments. However, these treatments are typically combined with pharmacological management to reduce pain, which can introduce additional unwanted consequences. Alternative treatments that rely on direct or indirect electrical stimulation of the spinal column have shown promise. SCS uses implantation of electrodes to delivery direct electrical stimulation to the spinal column.
Alternatively, PEMF therapy involves application of an electromagnetic field to the affected lumbar region. Unlike SCS, such an approach obviates the need for electrodes, thus is noninvasive, and can be self-administered.

In this article, we describe a pilot, double-blind, randomized clinical study that shows encouraging clinical results about the efficacy of PEMF therapy for the treatment of chronic pain associated with FBSS. This study investigated the use of PEMF therapy as an adjunctive intervention for postoperative pain in a defined group of subjects who had previously undergone back surgery (lower lumbar, nonfusion) and reported chronic back and/or radiating leg pain. The strength of the current study is the double-blind, shamcontrolled study design, which is not commonly incorporated in clinical interventional studies of FBSS. Whereas the focus was on the currently available active treatment (42- $\mu$ s group), a second exploratory active arm was added (38- $\mu$ s group). The primary outcome analysis evaluated the average percentage reduction in PI between baseline and end of treatment (Days 56-60). By this measure, the 42- $\mu$ s group reported a mean decrease of $40.2 \%$ in back PI at the end of treatment compared with a decrease of $25.6 \%$ in PI for the sham group 
(Table 3). The mean decrease in leg PI reported for the $42-\mu \mathrm{s}$ group was $45.0 \%$ compared with a decrease of $24.5 \%$ for the sham group. Moreover, results of responder, AUC, and time-to-event analyses were consistent with these findings. We readily acknowledge that the results of the prespecified analyses did not reach nominal statistical significance (ie, $p<0.05$ ); however, the sample was not powered for such differences. Nevertheless, several endpoints and alternative analyses demonstrated a consistent pattern indicative of a true treatment effect.

The decreases in pain scores were associated with modest decreases in analgesic use. It may be considered more relevant that the improvements in pain scores could not be attributed to any increase in self-administration of analgesics. Nevertheless, it is recognized that self-reporting of analgesic use and documentation of the reasons for such use can be challenging in clinical trials.

Even though it is not commonly used in FBSS trials, measurement of hsCRP may have some value as a quantitative tool for accessing pain in clinical trials for therapeutic treatments. Despite the applicability of hsCRP in lumbar pain trials being not well established, the decrease in the hsCRP levels in the $42-\mu$ s group suggest that this marker could be of value in assessing the effectiveness of therapeutic products for pain relief. ${ }^{33,34}$

Surprisingly, a small reduction in the dose of PEMF energy appeared to be associated with a diminution of the therapeutic effect. The decrease in pulse width to $38-\mu \mathrm{s}$ resulted in a decrease of PEMF dose by $10 \%$. This result underscores the need for further evaluation and optimization of PEMF therapy for the type of tissue and anatomical location being treated.

Another aspect of this study was to establish a placebo effect of PEMF therapy and medical devices in general. ${ }^{37-39}$ A true sham device is difficult to implement in the field of neuromodulation because of the nature of the therapy. Routinely, medical devices such as transcutaneous electrical nerve stimulation or SCS rely on conventional medical management as a control value in randomized controlled trials. The placebo effect may bring into question the interpretation of pain management therapies when a placebo cannot, or is not, used - especially in the treatment of chronic pain associated with FBSS. In this study, we found that the $42-\mu$ s group reported a moderately better outcome for PI improvement over placebo, although with the limitation that the trial included a small number of subjects and the results were not, in general, statistically significant.

As with other types of back pain, both nociceptive and neuropathic mechanisms may contribute to chronic pain associated with FBSS, depending on the underlying cause of an individual's pain symptoms., ${ }^{412}$ Whether continual stimulation of nociceptive pathways due to mechanical injury or prolonged peripheral sensory stimulation leading to neuropathic pain can cause chronic lumbar pain or FBSS is unclear. ${ }^{3,36}$ It has been suggested that, in some cases, FBSS could be the result of abnormal excitability of both the afferent or sensory neurons in the peripheral and central nervous systems. ${ }^{12}$

In terms of mechanism of action, one hypothesis is that PEMF may induce Eddy currents in biological tissue, which could in turn mediate downstream biological effects. Interventions involving electrical stimulation may inhibit pain in part by direct modulation of the nervous system, perhaps by stimulation of inhibitory sensory neurons as proposed in gate control theory, ${ }^{40,41}$ and/or mediate local electrochemical changes, ${ }^{24}$ which may, in turn, have downstream effects on gene expression. ${ }^{23,42-46}$ Recent evidence suggests that SCS and PEMF can mediate changes in gene expression, including genes implicated in pain pathways such as endogenous opioids and eicosanoid enzyme pathways. ${ }^{4243,45,46}$ Decreases in pain via a neuromodulation mechanism may explain, at least in part, improvements in radiating leg pain in this study and others. ${ }^{47,48}$ Attempts to use neuromodulation in the treatment of FBSS and neuropathy in general would benefit from a better understanding of the mechanism of action of treatment options that may mediate such an effect. Based on the results of this study, we hypothesize that specific PEMF energetics are effective for the treatment of chronic lumbar and radiating leg pain associated with FBSS.

This study had several primary limitations. Although this study was a double-blinded, randomized sham-controlled trial, the sample size was relatively small and changes did not show substantial differences between the groups. Nevertheless, trends were observed in favor of PEMF at a specific dose of $42 \mu \mathrm{s}$. Data from this study can be used as a basis for the design and sample size of future studies. A second limitation of this study was the time of treatment. Most studies conducted with medical devices that may affect neuromodulation (eg, SCS trials) cover a therapy period of 6-12 months with at least a 2-year follow-up. ${ }^{20,49,50}$ The lack of an extended follow-up limits the interpretation of whether PEMF therapy is actually resolving the cause of the pain or masking it. The last limitation relates to the aforementioned use of as-needed analgesics, which can confound pain score results; however, the as-needed use of pain medications reflects "real-life" practice and clinical care. 


\section{Conclusion}

The current study on subjects with persistent pain 3-36 months after lumbar surgery demonstrated consistent trends for a reduction in pain at the end of a 60-day treatment protocol with PEMF therapy compared with sham treatment. For subjects responding to the therapy, the time to $30 \%$ reduction in pain scores, $\mathrm{PGIC}$, and the reduction in the inflammatory marker hsCRP were consistent with a beneficial effect of PEMF over sham control. The potential influence of therapy dose was also shown, in that a $10 \%$ reduction in PEMF energy was associated with a possible diminution of therapeutic outcomes. In comparison with sham, this study supports the effectiveness of PEMF therapy for producing pain relief in patients with persistent pain following lumbar surgery. However, it is recognized that changes did not consistently exceed what is considered to be clinically meaningful. Although the results are encouraging, the findings should not be considered definitive. The data generated can be used to design larger trials with statistical power to confirm the efficacy of PEMF.

\section{Acknowledgments}

The authors acknowledge Dr Adrianne Smith, MD and Nicole Kubat, $\mathrm{PhD}$ for their help in drafting the article and critical review. The study was funded by Regenesis Biomedical Inc., Scottsdale, AZ, USA.

\section{Disclosure}

The authors report no conflicts of interest in this work.

\section{References}

1. Ragab A, Deshazo RD. Management of back pain in patients with previous back surgery. Am J Med. 2008;121(4):272-278.

2. Baber Z, Erdek MA. Failed back surgery syndrome: current perspectives. J Pain Res. 2016;9:979-987. eCollection 2016.

3. Chan CW, Peng P. Failed back surgery syndrome. Pain Med. 2011;12(4):577-606.

4. Rigoard P, Desai MJ, Taylor RS. Failed back surgery syndrome: what's in a name? A proposal to replace "FBSS" by "POPS". Neurochirurgie. 2015;61(Supp1 1):S16-S21.

5. Hussain A, Erdek M. Interventional pain management for failed back surgery syndrome. Pain Pract. 2014;14(1):64-78.

6. Assaker R, Zairi F. Failed back surgery syndrome: to re-operate or not to re-operate? A retrospective review of patient selection and failures. Neurochirurgie. 2015;61 Suppl 1:S77-S82.

7. Bordoni B, Marelli F. Failed back surgery syndrome: review and new hypotheses. J Pain Res. 2016;9:17-22.

8. Vleggeert-Lankamp CL, Arts MP, Jacobs WCh, Peul WC. Failed back (surgery) syndrome: time for a paradigm shift. Br J Pain. 2013;7(1):48-55.

9. Kumar K, North R, Taylor R, et al. Spinal cord stimulation vs. conventional medical management: a prospective, randomized, controlled, multicenter study of patients with failed back surgery syndrome (PROCESS study). Neuromodulation. 2005;8(4):213-218.
10. Leveque JC, Villavicencio AT, Bulsara KR, Rubin L, Gorecki JP. Spinal cord stimulation for failed back surgery syndrome. Neuromodulation. 2001;4(1):1-9.

11. North RB, Ewend MG, Lawton MT, Kidd DH, Piantadosi S. Failed back surgery syndrome: 5-year follow-up after spinal cord stimulator implantation. Neurosurgery. 1991;28(5):692-699.

12. Blond S, Mertens P, David R, Roulaud M, Rigoard P. From "mechanical" to "neuropathic" back pain concept in FBSS patients. A systematic review based on factors leading to the chronification of pain (part C). Neurochirurgie. 2015;61(Suppl 1):S45-S56.

13. Rigoard P, Assaker R. Failed back surgery syndrome: from pathophysiology to recent therapeutic advances in neurostimulation - introduction. Neurochirurgie. 2015;61(Suppl 1):S5.

14. Rajaee SS, Bae HW, Kanim LE, Delamarter RB. Spinal fusion in the United States: analysis of trends from 1998 to 2008. Spine (Phila Pa 1976). 2012;37(1):67-76.

15. Haanpää ML, Gourlay GK, Kent JL, et al. Treatment considerations for patients with neuropathic pain and other medical comorbidities. Mayo Clin Proc. 2010;85(3 Suppl):S15-S25.

16. Lee M, Silverman SM, Hansen H, Patel VB, Manchikanti L. A comprehensive review of opioid-induced hyperalgesia. Pain Physician. 2011;14(2):145-161.

17. Manchikanti L, Benyamin R, Datta S, Vallejo R, Smith H. Opioids in chronic noncancer pain. Expert Rev Neurother. 2010;10(5):775-789.

18. Chou R, Huffman LH. Guideline for the Evaluation and Management of Low Back Pain: Evidence Review. Glenview, IL: American Pain Society; 2007.

19. Sirohi S, Tiwari AK. Pain in the management of opioid use disorder. $J$ Pain Res. 2016;9:963-966. eCollection 2016.

20. Grider JS, Manchikanti L, Carayannopoulos A, et al. Effectiveness of spinal cord stimulation in chronic spinal pain: a systematic review. Pain Physician. 2016;19(1):E33-E54.

21. Verrills P, Sinclair C, Barnard A. A review of spinal cord stimulation systems for chronic pain. J Pain Res. 2016;9:481-492.

22. Harper WL, Schmidt WK, Kubat NJ, Isenberg RA. An open-label pilot study of pulsed electromagnetic field therapy in the treatment of failed back surgery syndrome pain. Int Med Case Rep J. 2014;8:13-22.

23. Guo L, Kubat NJ, Isenberg RA. Pulsed radio frequency energy (PRFE) use in human medical applications. Electromagn Biol Med. 2011;30(1):21-45.

24. Malmivuo J, Plonsey R. Electric and magnetic stimulation of neural tissue. In: Malmivuo J, Plonsey R, editors. Bioelectromagnetism: Principles and Applications of Bioelectric and Biomagnetic Fields. New York: Oxford University Press; 1995:363-380.

25. Guo L, Kubat NJ, Nelson TR, Isenberg RA. Meta-analysis of clinical efficacy of pulsed radio frequency energy treatment. Ann Surg. 2012;255(3):457-467.

26. Farrar JT, Polomano RC, Berlin JA, Strom BL. A comparison of change in the $0-10$ numeric rating scale to a pain relief scale and global medication performance scale in a short-term clinical trial of breakthrough pain intensity. Anesthesiology. 2010;112(6):1464-1472.

27. Farrar JT, Young JP Jr, LaMoreaux L, Werth JL, Poole RM. Clinical importance of changes in chronic pain intensity measured on an 11-point numerical pain rating scale. Pain. 2001;94(2):149-158.

28. Pruessner JC, Kirschbaum C, Meinlschmid G, Hellhammer DH. Two formulas for computation of the area under the curve represent measures of total hormone concentration versus time-dependent change. Psychoneuroendocrinology. 2003;28(7):916-931.

29. Dworkin RH, Turk DC, Wyrwich KW, et al. Interpreting the clinical importance of treatment outcomes in chronic pain clinical trials: IMMPACT recommendations. J Pain. 2008;9(2):105-121.

30. Scott W, McCracken LM. Patients' impression of change following treatment for chronic pain: global, specific, a single dimension, or many? J Pain. 2015;16(6):518-526.

31. Fairbank JC, Pynsent PB. The Oswestry Disability Index. Spine (Phila Pa 1976). 2000;25(22):2940-2952; discussion 2952. 
32. Beck AT, Ward CH, Mendelson M, Mock J, Erbaugh J. An inventory for measuring depression. Arch Gen Psychiatry. 1961;4:561-571.

33. Park $\mathrm{CH}$, Lee SH. Investigation of high-sensitivity C-reactive protein and erythrocyte sedimentation rate in low back pain patients. Korean J Pain. 2010;23(2):147-150.

34. Rathod TN, Chandanwale A, Ladkat KM, Chavan S, Chavan A, Bhosale PB. High sensitive C-reactive protein-effective tool in determining postoperative recovery in lumbar disc disease. Indian J Orthop. 2014;48(4):354-359.

35. Hayek SM, Helm S, Benyamin RM, Singh V, Bryce DA, Smith HS. Effectiveness of spinal endoscopic adhesiolysis in post lumbar surgery syndrome: a systematic review. Pain Physician. 2009;12(2): 419-435.

36. Markman JD, Kress BT, Frazer M, Hanson R, Kogan V, Huang JH. Screening for neuropathic characteristics in failed back surgery syndromes: challenges for guiding treatment. Pain Med. 2015;16(3): 520-530.

37. Brody H. Placebos and the Philosophy of Medicine: Clinical, Conceptual, and Ethical Issues. Chicago, IL: University of Chicago Press; 1980.

38. Conrad R. The hardest thing to see is what is in front of your eyes quo vadis placebo analgesia? J Pain Res. 2016;9:819-823. eCollection 2016.

39. Vase L, Petersen GL, Riley JL 3rd, Price DD. Factors contributing to large analgesic effects in placebo mechanism studies conducted between 2002 and 2007. Pain. 2009;145(1-2):36-44.

40. Melzack R, Wall PD. Pain mechanisms: a new theory. Science. 1965;150(3699):971-979.

41. Moayedi M, Davis KD. Theories of pain: from specificity to gate control. J Neurophysiol. 2013;109(1):5-12.

42. Kubat NJ, Moffett J, Fray LM. Effect of pulsed electromagnetic field treatment on programmed resolution of inflammation pathway markers in human cells in culture. J Inflamm Res. 2015;8:59-69.
43. Moffett J, Fray LM, Kubat NJ. Activation of endogenous opioid gene expression in human keratinocytes and fibroblasts by pulsed radiofrequency energy fields. J Pain Res. 2012;5:347-357.

44. Shanthanna H, Chan P, McChesney J, Paul J, Thabane L. Assessing the effectiveness of 'pulse radiofrequency treatment of dorsal root ganglion' in patients with chronic lumbar radicular pain: study protocol for a randomized control trial. Trials. 2012;13:52.

45. Tilley DM, Cedeño DL, Kelley CA, Benyamin R, Vallejo R. Spinal cord stimulation modulates gene expression in the spinal cord of an animal model of peripheral nerve injury. Reg Anesth Pain Med. 2016;41(6):750-756.

46. Vallejo R, Tilley DM, Cedeño DL, Kelley CA, DeMaegd M, Benyamin R. Genomics of the effect of spinal cord stimulation on an animal model of neuropathic pain. Neuromodulation. 2016;19(6):576-586.

47. Kumar K, Taylor RS, Jacques L, et al. Spinal cord stimulation versus conventional medical management for neuropathic pain: a multicentre randomised controlled trial in patients with failed back surgery syndrome. Pain. 2007;132(1-2):179-188

48. North RB, Kidd DH, Farrokhi F, Piantadosi SA. Spinal cord stimulation versus repeated lumbosacral spine surgery for chronic pain: a randomized, controlled trial. Neurosurgery. 2005;56(1):98-106; discussion 106-107.

49. Kapural L, van Buyten JP, Verrills P. Response: high frequency (10 $\mathrm{kHz}$ ) or burst spinal cord stimulation in failed back surgery syndrome patients with predominant back pain: preliminary data from a prospective observational study. Neuromodulation. 2016;19(7):787.

50. Kumar K, Hunter G, Demeria D. Spinal cord stimulation in treatment of chronic benign pain: challenges in treatment planning and present status, a 22-year experience. Neurosurgery. 2006;58(3):481-496; discussion 481-496.

51. Tennant F, Sajben N, Mack J, Stall RS. Interpreting indications for electromagnetic therapy. Pract Pain Manag. 2013;13(5):6-7; Consensus Statement 7. 


\section{Supplementary material}

Table SI Titles of participating sites:

01 - AOC Research, Birmingham, AL, USA [Dr R Sorrell]

02 - Injury Care, Boise, ID, USA [Dr R Radnovich]

03 - Danville Orthopedic Clinic, Danville, VA, USA [Dr L Abram]

04 - Upstate Clinical Trials, Spartanburg, SC, USA [Dr Y Mironer]

05 - Coastal Orthopedics, Bradenton, FL, USA [Dr R Bundschu]

06 - Tarheel Clinical Research, Raleigh, NC, USA [Dr W Harper]

07 - Millennium Pain Center, Bloomington, IL, USA [Dr R Benyamin]

08 - HOPE Research Institute, Phoenix, AZ, USA [Dr M Doust]

09 - HOPE Research Institute, Las Vegas, NV, USA [Dr W Le]

10 - Navarro Research Group, Chula Vista, CA, USA [Dr R Navarro]

II - Washington Center for Pain Management, Everett, WA, USA

[Dr P Mambalam]

12 - Physicians' Research Options (PRO), Sandy, UT, USA [Dr A Krull]

13 - Physicians' Research Options (PRO), Las Vegas, NV, USA [Dr W Muir]

The Journal of Pain Research is an international, peer reviewed, open access, online journal that welcomes laboratory and clinical findings in the fields of pain research and the prevention and management of pain. Original research, reviews, symposium reports, hypothesis formation and commentaries are all considered for publication.

\section{Dovepress}

The manuscript management system is completely online and includes a very quick and fair peer-review system, which is all easy to use. Visit http://www.dovepress.com/testimonials.php to read real quotes from published authors. 